\title{
A molecular framework for light and gibberellin control of cell elongation
}

\author{
Miguel de Lucas ${ }^{1 *}$, Jean-Michel Davière ${ }^{1 *}$, Mariana Rodríguez-Falcón ${ }^{1 *}$, Mariela Pontin ${ }^{1}$, \\ Juan Manuel Iglesias-Pedraz ${ }^{1}$, Séverine Lorrain ${ }^{2}$, Christian Fankhauser ${ }^{2}$, Miguel Angel Blázquez ${ }^{3}$, \\ Elena Titarenko ${ }^{1} \&$ Salomé Prat ${ }^{1}$
}

Cell elongation during seedling development is antagonistically regulated by light and gibberellins (GAs) ${ }^{1,2}$. Light induces photomorphogenesis, leading to inhibition of hypocotyl growth, whereas GAs promote etiolated growth, characterized by increased hypocotyl elongation. The mechanism underlying this antagonistic interaction remains unclear. Here we report on the central role of the Arabidopsis thaliana nuclear transcription factor PIF4 (encoded by PHYTOCHROME INTERACTING FACTOR $4)^{3}$ in the positive control of genes mediating cell elongation and show that this factor is negatively regulated by the light photoreceptor phyB (ref. 4) and by DELLA proteins that have a key repressor function in GA signalling ${ }^{5}$. Our results demonstrate that PIF4 is destabilized by phyB in the light and that DELLAs block PIF4 transcriptional activity by binding the DNA-recognition domain of this factor. We show that GAs abrogate such repression by promoting DELLA destabilization, and therefore cause a concomitant accumulation of free PIF4 in the nucleus. Consistent with this model, intermediate hypocotyl lengths were observed in transgenic plants over-accumulating both DELLAs and PIF4. Destabilization of this factor by phyB, together with its inactivation by DELLAs, constitutes a protein interaction framework that explains how plants integrate both light and GA signals to optimize growth and development in response to changing environments.

Seedlings undergo alternative developmental programmes depending on whether they are germinated in the dark or in the light. Dark-grown seedlings exhibit etiolated growth, characterized by long hypocotyls, small and closed cotyledons with undifferentiated chloroplasts, and the repression of light-regulated genes ${ }^{1}$. During photomorphogenesis, light inhibits hypocotyl growth and promotes cotyledon opening and expansion, chloroplast differentiation and the activation of light-regulated genes. phyB is the main photoreceptor mediating de-etiolation in red light ${ }^{4,6}$. Absorption of red light converts this photoreceptor into a Pfr active form that is translocated into the nucleus ${ }^{7,8}$; Pfr interacts there with members of the bHLH family of phytochrome-interacting factors (PIFs), involved in modulation of light-regulated genes with a role in photomorphogenesis ${ }^{1,4}$.

Gibberellins (GAs) exert an opposite effect to light on photomorphogenesis ${ }^{2}$. GAs promote etiolated growth, whereas GA-deficiency induces a partially de-etiolated phenotype in the dark, which is reverted by a lack of DELLA function ${ }^{2,9}$. DELLAs function as key repressors of GA-responsive growth, by inhibiting GA-regulated gene expression ${ }^{5}$. These repressors accumulate in the nucleus and are rapidly degraded in response to $\mathrm{GA}^{10,11}$. In Arabidopsis, RGA (encoded by repressor of ga1-3) and GAI (encoded by GA insensitive) are the main repressors controlling hypocotyl growth and stem elongation $^{12,13}$. Mutations within the DELLA domain render these proteins resistant to degradation, and result in a GA-insensitive dwarf phenotype $\mathrm{e}^{12,14}$. This domain binds the GA receptor GID1 (ref. 15) in a GA-dependent manner, which promotes interaction with the F-box protein SLEEPY1 (SLY1) and polyubiquitination of these proteins by the SCF ${ }^{\text {SLY1/GID2 }}$ ligase complex, thereby signalling their degradation by the $26 \mathrm{~S}$ proteasome pathway ${ }^{16,17}$.

The functional mechanism by which DELLAs regulate gene expression and promote photomorphogenesis remains unclear. Attempts to demonstrate direct DNA-binding ability of DELLAs have been unsuccessful, indicating that these repressors might exert their negative regulatory function through protein-protein interaction with other transcription factors. Here we report on the interaction of DELLAs with the PIF4 and PIF3 bHLH factors using a yeast two-hybrid-based screen (Fig. 1a and Supplementary Fig. 1), and provide evidence for a crucial role of these factors in the integration of both GA and light signals to modulate hypocotyl growth.

Pull-down assays using a purified glutathione S-transferase (GST)RGA protein confirmed interaction of DELLAs with the PIF4 and PIF3 factors and showed an increased affinity for PIF4 binding (Fig. 1b). Bimolecular fluorescence complementation (BiFC) assays in Nicotiana benthamiana leaves demonstrated interaction of these proteins in living plant cells (Fig. 1c). In addition, co-immunoprecipitation studies using transgenic green fluorescent protein (GFP)-RGA lines ${ }^{10}$ further corroborated this interaction (Fig. 1d). Co-immunoprecipitation with an anti-GFP antibody and subsequent detection with an antibody raised against the PIF4 protein showed that binding to the RGA repressor is more efficient in seedlings treated with the inhibitor of GA biosynthesis paclobutrazol (PAC), which induces RGA accumulation, and also in seedlings exposed to dark (see Fig. 1d). Treatment with GA promotes RGA degradation and abolishes this interaction (Fig. 1d).

The pif4 mutant has short hypocotyls in red and white light, whereas PIF4 overexpressors (35S-PIF4) show a long-hypocotyl phenotype that resembles the phyB mutants ${ }^{3}$. We also demonstrate that these plants have an altered response to PAC and GA treatments, indicative of a PIF4 role in GA-induced hypocotyl growth control. Response to increasing concentrations of PAC was reduced in $p h y B$ and 35S-PIF4 (in an slr2 background) seedlings, whereas pif4 seedlings showed a hypersensitive response to this inhibitor (Fig. 2a, c). GA application, in turn, induced an exaggerated elongation response in $p h y B$ and 35S-PIF4 seedlings, whereas pif4 was partially insensitive to this treatment (Fig. $2 \mathrm{~b}, \mathrm{c}$ ). These results thus point to a possible

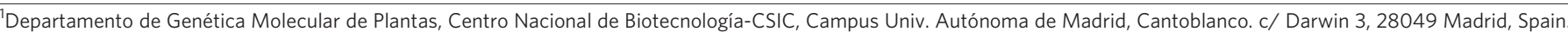

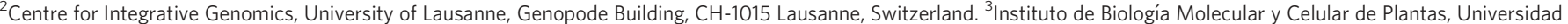
Politécnica de Valencia, Consejo Superior de Investigaciones Científicas, 46022 Valencia, Spain.

*These authors contributed equally to this work. 
function of this transcription factor as an integration node for both the light and GA pathways.

Deletion studies revealed that the PIF4 bHLH DNA-recognition domain is responsible for interaction of this factor with RGA (Supplementary Fig. 2a). In addition, we determined that the first conserved heptad leucine repeat in the RGA protein mediates interaction of this repressor with PIF4 (Supplementary Fig. 2b). Of note, this heptad repeat region is highly conserved in all members of the DELLA family and an interaction between PIF4 with the GAI, RGL1 or RGL3 repressors is also observed in yeast cells (Supplementary Fig. 3). Identification of the PIF4 bHLH domain as the domain mediating interaction with the DELLAs raised the possibility that these repressors may block PIF4 DNA-binding ability. To test this possibility, we performed transient expression assays, using a GUS ( $\beta$-glucuronidase gene) fusion to the LTP3 promoter (At5g59320) as a reporter for PIF4 transcriptional activity. Effector constructs for the PIF4 and RGA proteins, and for deletion derivatives of the DELLA repressor resistant to GA destabilization $(\triangle \mathrm{RGA})$ or those unable to interact with PIF4 (del1RGA), were expressed under control of the $2 \times C a M V$ $35 S$ promoter and co-bombarded together with the reporter construct into Arabidopsis thaliana cells. As seen in Fig. 2d, expression of PIF4 resulted in a 2.6-fold stimulation of the LTP3 reporter activity, providing evidence for a positive regulatory activity of this factor. Co-expression of PIF4 and the RGA or $\triangle \mathrm{RGA}$ proteins strongly repressed LTP3 expression, demonstrating that these repressors block PIF4 transcriptional activity. More importantly, treatment with GA suppressed the inhibitory effect of RGA by triggering degradation of the protein. However, the stable $\triangle \mathrm{RGA}$ protein lacking the DELLA domain was unresponsive to this treatment (Fig. 2d). Coexpression of del1RGA, in turn, did not suppress PIF4 transcriptional activity (Fig. 2d), demonstrating that the observed repression is dependent on RGA-PIF4 interaction. Hence, these results are congruent with a positive regulatory function of PIF4 in cell elongation, and with DELLAs repressing PIF4 activity by forming an inactive complex with this factor. a

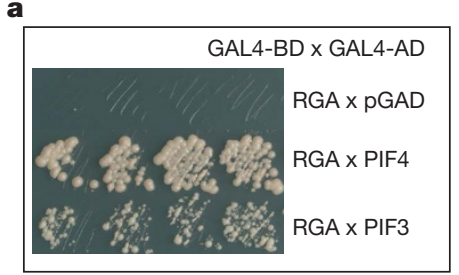

c YFP $^{\mathrm{N}}-\mathrm{PIF} 4+\mathrm{RGA}-\mathrm{YFP}^{\mathrm{C}}$

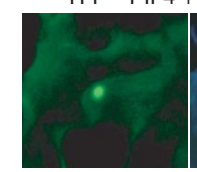

YFP

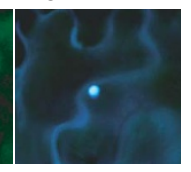

DAPI

d

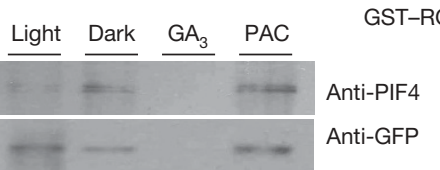

b

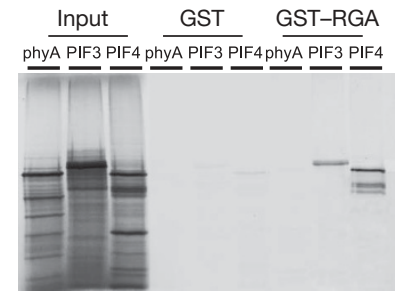

Figure 1 | DELLA-PIF4 interaction in yeast two-hybrid and pull-down assays and interaction of these proteins in Nicotiana benthamiana leaves and Arabidopsis thaliana seedlings. a, Interaction between DELLA and the PIF4 and PIF3 bHLH factors in - His - Ade plates. BD, binding domain; $\mathrm{AD}$, activation domain. $\mathbf{b}$, Pull-down assays showing the interaction between RGA and the PIF3 and PIF4 factors. Quantification of the radio-labelled pulled down phyA, PIF3 and PIF4 proteins is shown. c, BiFC analysis of PIF4-DELLA interaction. YFP, eYFP fluorescence; DAPI, 4,6-diamidino-2phenylindole nuclei staining. d, Co-immunoprecipitation of the Arabidopsis GFP-RGA and PIF4 proteins. Plant extracts were immunoprecipitated with an anti-GFP antibody and detected by western blot using an antibody raised against PIF4 and the anti-GFP antibody.
The observation that the $p h y B$ hypocotyl phenotype is epistatic to pif4 led to the proposal that PIF4 acts as a negative regulator of phyB signalling ${ }^{3}$. However, consistent with recent reports showing an additive function of PIF4 and PIF5 in hypocotyl elongation ${ }^{18-20}$, we found that an additional mutation of the PIF5 gene suppresses the
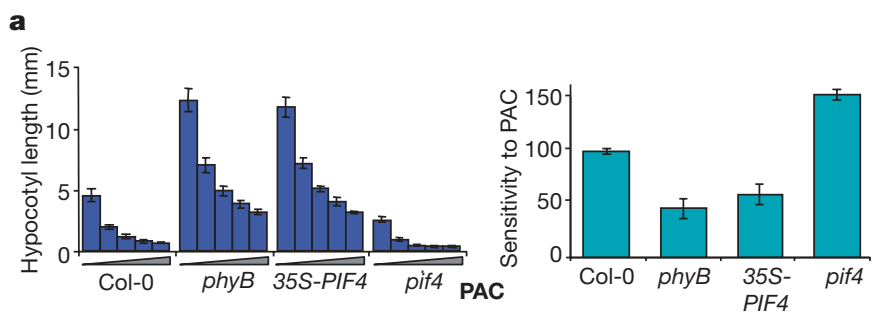

b

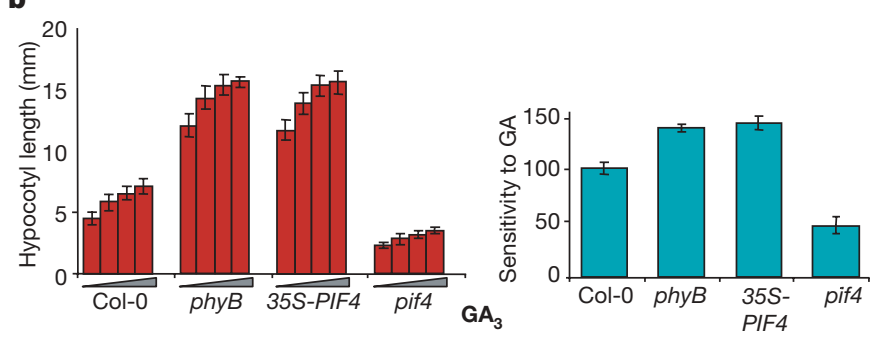

c

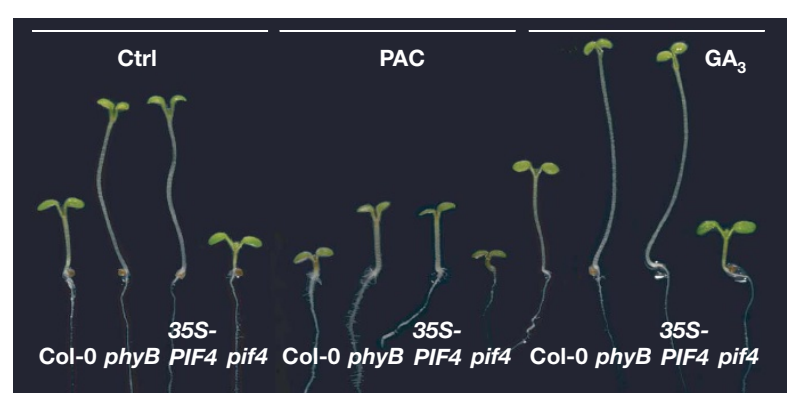

d
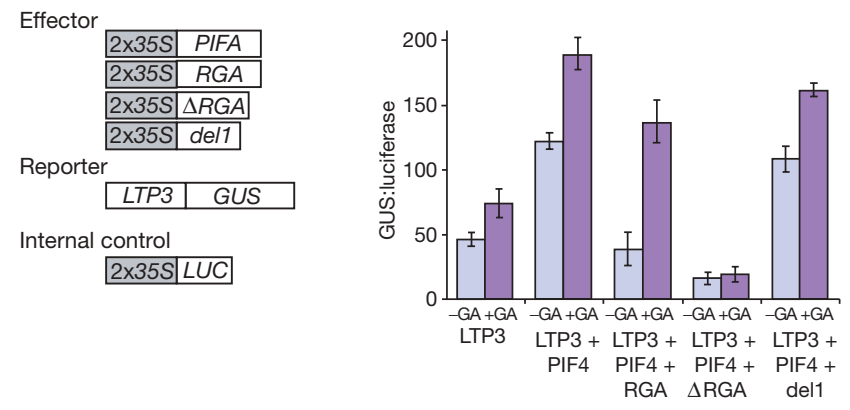

Figure 2 | Altered response to GA and paclobutrazol (PAC) treatments of 35S-PIF4 and pif4 lines, and transient expression assays providing evidence of a block of PIF4 transcriptional activity by DELLAs. a, Hypocotyl lengths of Col-0, phyB, 35S-PIF4 and pif4 seedlings grown in the presence of increasing concentrations $(0,0.025,0.05,0.1$ and $0.2 \mu \mathrm{M})$ of the GA biosynthesis paclobutrazol (PAC) inhibitor. Right panel, relative response to PAC treatment. Means \pm s.d. were obtained from 20 independent plants. Values are the mean of three independent experiments; error bars, s.d. between experiments. $\mathbf{b}$, Hypocotyl lengths of seedlings grown under increasing concentrations of $\mathrm{GA}_{3}(0,2.5,5.0$ and $10 \mu \mathrm{M})$. Right panel, relative response to GA treatment. Means \pm s.d. were obtained from 20 independent plants; error bars are as before. c, Phenotypes of the PAC- and GA-treated seedlings. d, Transient assays of PIF4 transcriptional activity in Arabidopsis cells. Constructs used in the experiment are shown on the left. Cells were bombarded with the LTP3-GUS reporter alone or co-bombarded with combinations of these constructs, as indicated. A 35S-LUC (luciferase) construct was used as the internal control for transformation. Cells were incubated without ( $-\mathrm{GA}$ ) or with $50 \mu \mathrm{M}(+\mathrm{GA}) \mathrm{GA}_{3}$ and transcriptional activity measured as the ratio of GUS:LUC activity. Histogram columns represent the mean of eight biological replicates; error bars, s.e.m. 
elongated phenotype of phyB pif4 seedlings (Supplementary Fig. 4a, b). Hypocotyl growth of these lines correlates with levels of expression of transcripts LTP3 (At5g59320) and $\beta$-expansin (At2g20750), found to be upregulated in phyB and 35S-PIF4 seedlings and repressed in the pif4 mutant (Supplementary Fig. 4c). Further reduction of these transcripts in the pif4 pif5 mutant confirmed an additive function of these factors. Interestingly, phyB pif4 pif5 mutants remained taller than pif4 pif5 seedlings, indicating that phyBregulated factors other than PIF4 and PIF5 might also participate in hypocotyl growth control (see ref. 21).

A positive regulatory function of PIF4 was further supported by chromatin immuno-precipitation (ChIP) assays using lines expressing a fusion of PIF4 to the haemagglutinin (HA) antigen (PIF4HA). PCR amplification of the upstream regions of genes differentially expressed in the 35 S-PIF4 or pif4 pif5 mutant lines showed that PIF4-HA bound exclusively the upstream region of upregulated genes with a G-box element in their promoters (Fig. 3a). Induced

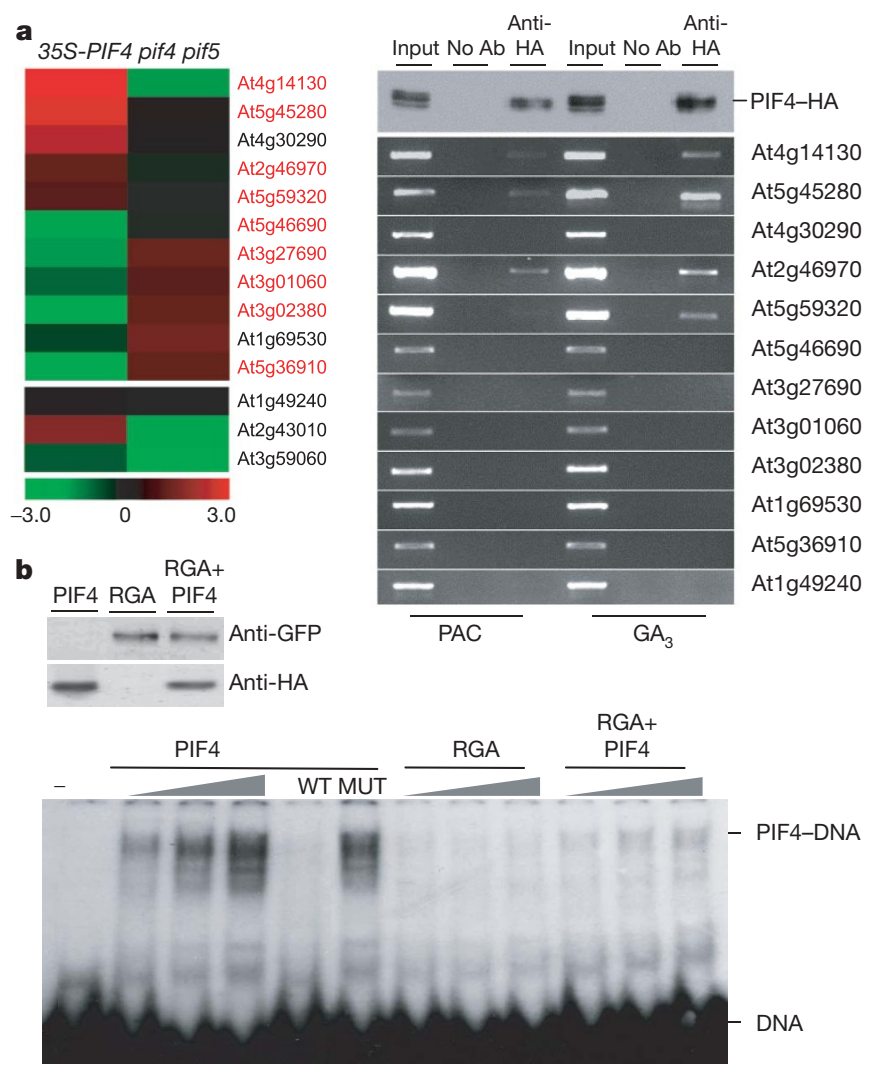

Figure 3 | Positive regulatory function of PIF4 and block of PIF4 DNAbinding ability by the DELLAs. a, Chromatin immunoprecipitation and promoter PCR amplification analysis, using PIF4-HA seedlings. Left panel, levels of expression of the selected genes in 35S-PIF4 and pif4 pif5 lines.

Colour scale represents fold-change (log). Genes exhibiting a G-box in their promoters are written in red. Right panel, PCR amplification of the immunoprecipitated PIF4-DNA complexes. Anti-HA indicates immunoprecipitation with an anti-HA antibody. Samples processed equally but without antibody (no $\mathrm{Ab}$ ) were used as negative control. Seedlings were incubated overnight with $0.1 \mu \mathrm{M}$ PAC or $25 \mu \mathrm{M} \mathrm{GA}_{3}$ (GA) in the dark. Western detection was used to assess that similar amounts of the PIF4-HA protein (PIF4-HA) were recovered in both treatments. b, EMSA studies using an LTP3 promoter fragment with a G-box element. N. benthamiana leaves infiltrated with the PIF4-HA and RGA-GFP constructs, or a 1:1 mixture of these Agrobacterium strains, were used to obtain the proteins. The abundance of the PIF4 and RGA proteins in these extracts was evaluated by western blot with anti-HA (PIF4) and anti-GFP (RGA) antibodies. The LTP3 DNA probe was incubated with increasing amounts of the proteins as indicated. -, incubation without protein extract. WT and MUT, competition with a 100-fold excess of cold wild-type (WT) and mutated (MUT) probes. genes lacking a G-box or those corresponding to repressed genes were not amplified in these assays, demonstrating that these genes may be secondary targets of PIF4 activity. Interaction of PIF4 with its targets was strongly reduced in seedlings accumulating the DELLA repressors (PAC treated), whereas it was enhanced in seedlings treated with GAs to destabilize the DELLAs (Fig. 3a). Hence, these results provided experimental evidence for a role of DELLAs in blocking PIF4 DNA-binding ability in vivo. Additional evidence for such a sequestration mechanism was obtained by electrophoretic mobility-shift assay (EMSA) experiments using protein extracts of Nicotiana benthamiana leaves agroinfiltrated with the PIF4-HA or GFP-RGA constructs, or with a 1:1 mix of these Agrobacterium strains. Indeed, PIF4-HA bound an LTP3 promoter fragment containing a G-box but co-expression of GFP-RGA abolished such binding activity (Fig. 3b) although it did not affect PIF4-HA levels (see Fig. 3b). Competition experiments with the cold probes showed that this binding activity requires an intact G-box.

Overall, our results are consistent with a positive-control function of PIF4 on hypocotyl growth and indicate that phyB signalling might repress hypocotyl growth by inhibiting PIF4 transcriptional activity. Nuclear translocation of phytochromes was in fact reported to induce proteasome-mediated degradation of the PIF3 and PIF1/ PIL5 factors ${ }^{22,23}$. Therefore it is possible that phyB exerts a similar control on PIF4. Our experiments indeed established that PIF4-GFP nuclear fluorescence was high in etiolated seedlings but rapidly disappeared on light irradiation (Fig. 4a). Seedling pre-treatment with the 26S proteasome inhibitor MG132 stabilized the protein in the light and, more importantly, light-dependent destabilization of the protein was not observed in $p h y B$ mutant seedlings, showing that phyB signalling is required for proteasome-mediated degradation of PIF4 (Fig. 4). Similar results have been reported recently for PIF5, corroborating our findings ${ }^{24}$.

The functional significance of these results was further investigated by overexpressing the PIF4 factor in a 20-oxidase GA-deficient background $(200 x)$ or in seedlings expressing the gai dominant allele (35S-gai), which lacks the DELLA domain and confers a GAinsensitive phenotype ${ }^{13}$. PIF4 overexpression in the $200 x$ or gai mutants resulted in notable hypocotyl growth compared to the mutations alone (Fig. 4b), demonstrating that this transcription factor is able to rescue partly the growth restraint imposed by DELLA accumulation. GA treatment, in addition, fully restored growth of 35S-PIF4 200x hypocotyls by inducing DELLA destabilization, but it did not alter growth of 35S-PIF4 gai seedlings, which accumulate a stable form of these repressors (Fig. 4b).

Collectively, our data are indicative of a positive function of the PIF4 and PIF5 factors in activated expression of cell elongation genes. In the light, phyB negatively regulates PIF4 transcriptional activity, by targeting degradation of this transcription factor by the $26 \mathrm{~S}$ proteasome pathway (Fig. 4c). DELLAs repress transcriptional activity of the PIF factors by interacting with the bHLH DNA-recognition domain and sequestering these factors into an inactive complex, unable to bind DNA (Fig. 4c). Consistent with this mode of action, stabilization of the DELLA proteins represses PIF4-mediated cell growth, whereas GAs induce elongation growth by destabilizing these repressors, allowing accumulation of free PIF4 in the nucleus and the activation of PIF4-regulated genes (Fig. 4c). Inactivation of this PIF factor by DELLA protein interaction or by phyB-mediated destabilization actually explains the intermediate hypocotyl lengths of double phyB ga1.3, phyB ga4, or phyB gai mutants ${ }^{25}$ or those of the transgenic 35S-PIF4 20ox and 35S-PIF4 gailines (Fig. 4b), which were not previously understood in the context of a simple genetic pathway.

PIFs are members of a subfamily of bHLH proteins with highly related DNA-binding domains and it is therefore possible that DELLAs block transcriptional activity of all members of this gene family. DELLA repressor interaction with PIF3 is in fact described in a companion report ${ }^{21}$. Notably, whereas PIF3 and PIF4 primary function is in hypocotyl elongation control ${ }^{3,26}$, other PIFs have been 
a

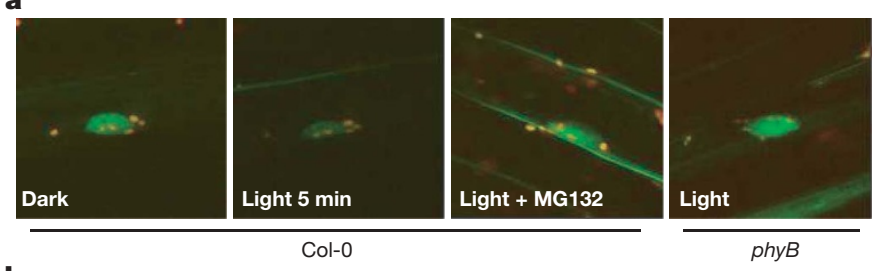

b
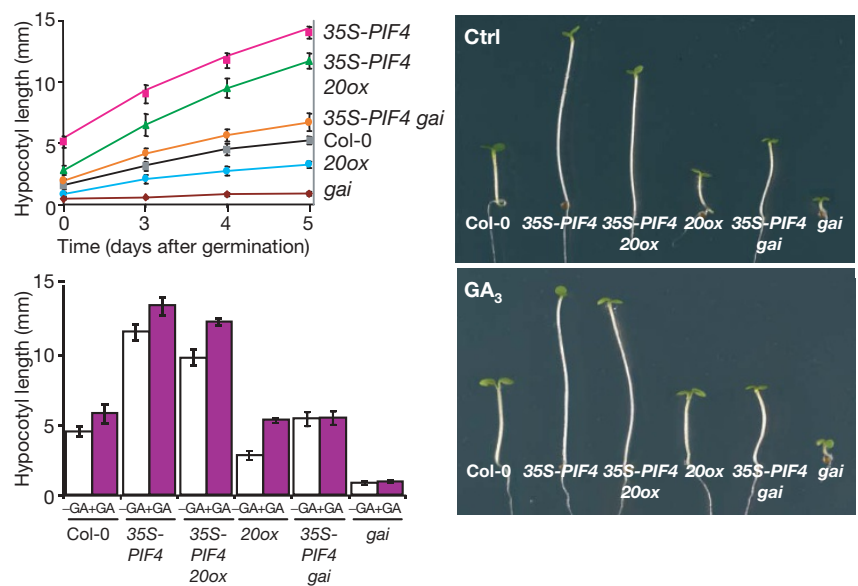

C
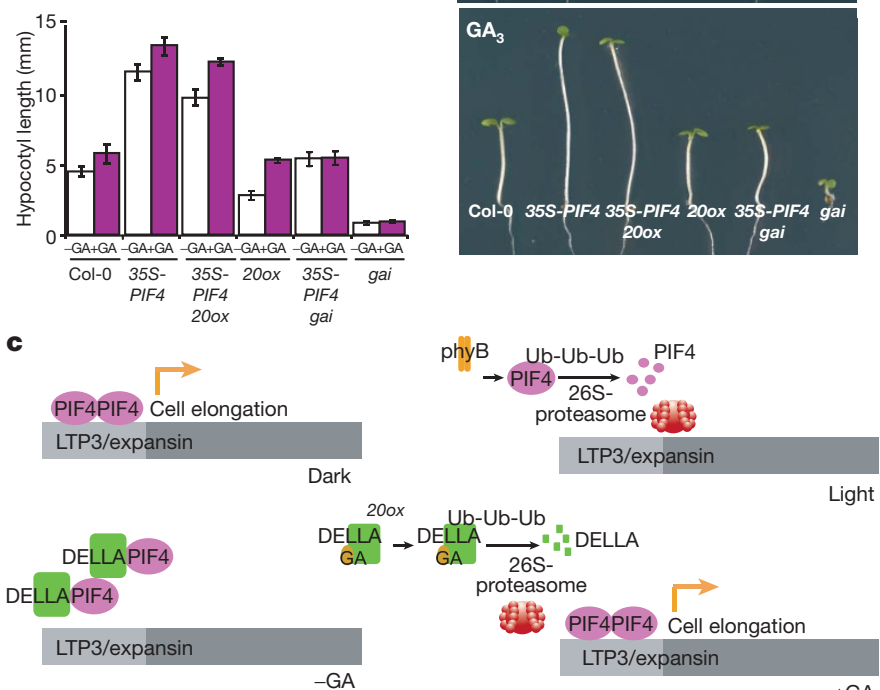

Figure 4 | PHYB-mediated degradation of PIF4 and intermediate hypocotyl lengths of 35S-PIF4 200x and 35S-PIF4 gai seedlings. a, Confocal fluorescence of nuclei of Col-0 and $p h y B$ transgenic lines expressing the PIF4-GFP construct. Seedlings were incubated overnight in the dark and GFP fluorescence visualized either directly (dark) or after 5 min of irradiation with white light (light $5 \mathrm{~min}$ ). Light induced a decline in GFP fluorescence in Col-0 lines but not in the phyB mutant ( $p h y B)$. Treatment with the MG132 proteasome inhibitor stabilized GFP fluorescence of Col-0 seedlings in the light (light + MG132). $\mathbf{b}$, Hypocotyl lengths of the double 35S-PIF4 20ox and 35S-PIF4 gai lines. Accumulation of DELLAs in these lines leads to intermediate hypocotyl phenotypes. Treatment with $\mathrm{GA}_{3}$ rescued hypocotyl growth in the PIF4-OE 20ox lines but did not affect growth of 35S-PIF4 gai seedlings, accumulating a stable DELLA. Hypocotyl lengths at different days of germination (upper left panel) and growth response induced by $2.5 \mu \mathrm{M} \mathrm{GA}_{3}$ (lower left panel; phenotypes in right panels). Values represent the mean of 10 plants; error bars, s.d. c, Model for direct PIF4 integration of both light and GA signals. In the light, phyB induces destabilization of PIF4. DELLAs interact with PIF4 and repress its DNA-binding ability. GAs trigger proteasome degradation of the DELLA repressors and allow accumulation of free PIF4, promoting PIF4-activated gene expression.

reported to control other light-regulated responses such as chlorophyll biosynthesis ${ }^{27}$ or seed germination ${ }^{28}$ - processes that are also known to be modulated by DELLAs. Hence, competitive interaction with members of the PIF family of transcription factors might be a prevailing mechanism for DELLA function, serving to explain the great diversity of responses controlled by these repressors.

\section{METHODS SUMMARY}

Plant mutants and transgenic lines. Descriptions of mutants and transgenic lines used in this work is given in Methods. Double and triple mutations were genotyped using the primers indicated in Supplementary Table 1. The LTP3 promoter and the RGA, GAI, RGL1 and RGL3 genes were amplified from Col0 genomic DNA using the primer combinations listed in Supplementary Table 3. PIF4 was amplified from leaf RNA. Constructs and fusions to the GFP and enhanced YFP fluorescent proteins are described in Methods.

Protein interaction assays. The yeast GAL4 system was employed for twohybrid screening with DELLAs. For pull-down assays, a GST-RGA fusion bound to glutathione-Sepharose beads was incubated with ${ }^{35}$ S-labelled phyA, PIF3 and PIF4 proteins. BiFC assays were performed as described in Methods. For coimmunoprecipitation experiments, extracts of GFP-RGA seedlings were incubated with an anti-GFP antibody (Santa Cruz), immunoprecipitated with protein $\mathrm{G}$ agarose (Sigma) and analysed by western blot using an antibody raised against the PIF4 protein.

ChIP, transactivation and gel-shift assays. Chromatin immunoprecipitation (ChIP) was performed as described ${ }^{29}$. PIF4-HA seedlings and an anti-HA antibody (Santa Cruz) were used in these assays. Transient expression and gel-shift assays were performed as described ${ }^{30}$. Details for constructs and bombardment conditions are given in the Methods. A fragment of the LTP3 promoter (At5g59320) with a G-box was used as a DNA probe for retardation. Leaves agro-infiltrated with the PIF4-HA and GFP-RGA constructs were used to obtain the proteins.

Full Methods and any associated references are available in the online version of the paper at www.nature.com/nature.

\section{Received 28 September; accepted 28 November 2007.}

1. Chen, M., Chory, J. \& Fankhauser, C. Light signal transduction in higher plants. Annu. Rev. Genet. 38, 87-117 (2004).

2. Alabadi, D., Gil, J., Blazquez, M. A. \& Garcia-Martinez, J. L. Gibberellins repress photomorphogenesis in darkness. Plant Physiol. 134, 1050-1057 (2004).

3. Huq, E. \& Quail, P. H. PIF4, a phytochrome-interacting bHLH factor, functions as a negative regulator of phytochrome B signaling in Arabidopsis. EMBO J. 21, 2441-2450 (2002).

4. Quail, P. H. Photosensory perception and signalling in plant cells: new paradigms? Curr. Opin. Cell Biol. 14, 180-188 (2002).

5. Sun, T. P. \& Gubler, F. Molecular mechanism of gibberellin signaling in plants. Annu. Rev. Plant Biol. 55, 197-223 (2004)

6. Neff, M. M., Fankhauser, C. \& Chory, J. Light: an indicator of time and place. Genes Dev. 14, 257-271 (2000).

7. Nagy, F. \& Schafer, E. Control of nuclear import and phytochromes. Curr. Opin. Plant Biol. 3, 450-454 (2000).

8. Huq, E., Al-Sady, B. \& Quail, P. H. Nuclear translocation of the photoreceptor phytochrome $B$ is necessary for its biological function in seedling photomorphogenesis. Plant J. 35, 660-664 (2003).

9. Achard, P. et al. DELLAs contribute to plant photomorphogenesis. Plant Physiol. 143, 1163-1172 (2007).

10. Silverstone, A. L. et al. Repressing a repressor: gibberellin-induced rapid reduction of the RGA protein in Arabidopsis. Plant Cell 13, 1555-1566 (2001).

11. $\mathrm{Fu}, \mathrm{X}$. et al. Gibberellin-mediated proteasome-dependent degradation of the barley DELLA protein SLN1 repressor. Plant Cell 14, 3191-3200 (2002)

12. Dill, A., Jung, H. S. \& Sun, T. P. The DELLA motif is essential for gibberellin-induced degradation of RGA. Proc. Natl Acad. Sci. USA 98, 14162-14167 (2001).

13. Peng, J. et al. The Arabidopsis GAl gene defines a signaling pathway that negatively regulates gibberellin responses. Genes Dev. 11, 3194-3205 (1997).

14. Peng, J. et al. 'Green revolution' genes encode mutant gibberellin response modulators. Nature 400, 256-261 (1999).

15. Ueguchi-Tanaka, M., Nakajima, M., Motoyuki, A. \& Matsuoka, M. Gibberellin receptor and its role in gibberellin signaling in plants. Annu. Rev. Plant Biol. 58, 183-198 (2007)

16. McGinnis, K. M. et al. The Arabidopsis SLEEPY1 gene encodes a putative F-box subunit of an SCF E3 ubiquitin ligase. Plant Cell 15, 1120-1130 (2003).

17. Dill, A., Thomas, S. G., Hu, J., Steber, C. M. \& Sun, T. P. The Arabidopsis F-box protein SLEEPY1 targets gibberellin signaling repressors for gibberellin-induced degradation. Plant Cell 16, 1392-1405 (2004)

18. Fujimori, T., Yamashino, T., Kato, T. \& Mizuno, T. Circadian-controlled basic/ helix-loop-helix factor, PIL6, implicated in light-signal transduction in Arabidopsis thaliana. Plant Cell Physiol. 45, 1078-1086 (2004).

19. Khanna, R. et al. A novel molecular recognition motif necessary for targeting photoactivated phytochrome signaling to specific basic helix-loop-helix transcription factors. Plant Cell 16, 3033-3044 (2004).

20. Nozue, K. et al. Rhythmic growth explained by coincidence between internal and external cues. Nature 448, 358-361 (2007).

21. Feng, S. et al. Coordinated regulation of Arabidopsis thaliana development by light and gibberellins. Nature doi:10.1038/nature06448 (this issue).

22. Al-Sady, B., Ni, W., Kircher, S., Schafer, E. \& Quail, P. H. Photoactivated phytochrome induces rapid PIF3 phosphorylation prior to proteasome-mediated degradation. Mol. Cell 23, 439-446 (2006).

23. Oh, E. et al. Light activates the degradation of PIL5 protein to promote seed germination through gibberellin in Arabidopsis. Plant J. 47, 124-139 (2006). 
24. Shen, Y., Khanna, R., Carle, C. M. \& Quail, P. H. Phytochrome induces rapid PIF5 phosphorylation and degradation in response to red-light activation. Plant Physiol. 145, 1043-1051 (2007).

25. Peng, J. \& Harberd, N. P. Gibberellin deficiency and response mutations suppress the stem elongation phenotype of phytochrome-deficient mutants of Arabidopsis. Plant Physiol. 113, 1051-1058 (1997).

26. Kim, J. et al. Functional characterization of phytochrome interacting factor 3 in phytochrome-mediated light signal transduction. Plant Cell 15, 2399-2407 (2003).

27. Huq, E. et al. Phytochrome-interacting factor 1 is a critical bHLH regulator of chlorophyll biosynthesis. Science 305, 1937-1941 (2004)

28. Oh, E. et al. PIL5, a phytochrome-interacting bHLH protein, regulates gibberellin responsiveness by binding directly to the $\mathrm{GAI}$ and RGA promoters in Arabidopsis seeds. Plant Cell 19, 1192-1208 (2007).

29. Lee, J. et al. Analysis of transcription factor HY5 genomic binding sites revealed its hierarchical role in light regulation of development. Plant Cell 19, 731-749 (2007)

30. Boter, M., Ruiz-Rivero, O., Abdeen, A. \& Prat, S. Conserved MYC transcription factors play a key role in jasmonate signaling both in tomato and Arabidopsis. Genes Dev. 18, 1577-1591 (2004).

Supplementary Information is linked to the online version of the paper at www.nature.com/nature.

Acknowledgements We thank P. Quail for the yeast PIF3 construct and 35S-PIF4 and pif4 lines, and P. Hedden for the $200 x$ lines. We also acknowledge F. Parcy for providing the BiFC vectors, J. A. Jarillo for advice with light treatments and the use of the red light irradiation chamber and $D$. Alabadi for critical reading of the manuscript. We thank J. Paz-Ares for his helpful suggestions concerning this work. This work was supported by a grant from the Spanish Ministerio de Educación y Ciencia. Work from the C.F. group was supported by an SNF grant. M.d.L. is a recipient of a predoctoral fellowship from the Spanish M.E.C. M.R.-F. was supported by a grant from the University of Talca and M.P. by a pre-doctoral fellowship from the Spanish A.E.C.I. J.-M.D. is a recipient of a CSIC I3P post-doctoral contract and S.L. of a Roche post-doctoral fellowship.

Author Contributions M.d.L. performed the bimolecular complementation assays, EMSA and co-immunoprecipitation studies and analysed phyB-mediated destabilization of the PIF4 protein; he was also co-responsible for the transient expression experiments, together with E.T. J.-M.D. performed the expression analyses and the selection of double and triple mutant lines and the ChIP experiments, in collaboration with J.M.I.-P. M.R.-F. performed the two-hybrid screening experiments and pull-down assays and together with M.P. mapped the interacting domains in both proteins. S.L. and C.F. provided the PIF4-HA lines and an antibody raised against the PIF4 protein. M.A.B. provided the 35S-gai lines and helped with critical discussions on the work. S.P. designed experiments, supervised the work and wrote the manuscript.

Author Information Reprints and permissions information is available at www.nature.com/reprints. Correspondence and requests for materials should be addressed to S.P. (sprat@cnb.uam.es). 


\section{METHODS}

Plant materials and growth conditions. Wild-type and mutant plants used in this study were all in the Col-0 ecotypes with the exception of the RGA-GFP$R G A$ lines $^{10}$, which were in the Ler ecotype. phyB-9 seeds ${ }^{31}$ were obtained from the Arabidopsis Biological Resource Centre. PIF4 overexpresser lines and pif4 knockout mutations (slr2 mutant) were those described in ref. 3. Double pif 4 pif 5 mutants were obtained by crossing the pif4-101 T-DNA insertion allele and the SALK-087012 mutant ${ }^{18}$ with an insertion in the PIF5 gene. $200 x$ lines carrying a knockout T-DNA insertion in the AtGA20ox1 gene (At4g25420) were provided by P. Hedden. GA-insensitive gai lines expressed the stable GAI protein under control of the $35 S$ promoter. Double and triple mutants were generated by crossing these lines and genotyping the offspring by PCR amplification or northern blot analyses.

Seeds were surface-sterilized and sown on GM agar plates without sucrose ${ }^{32}$. Plates were cold-treated for $2 \mathrm{~d}$ at $4{ }^{\circ} \mathrm{C}$ and germination was synchronized by $3 \mathrm{~h}$ of irradiation with white light and subsequent incubation in the dark for $22 \mathrm{~h}$, before transfer to the different growth conditions. White-light-grown seedlings were grown at $20^{\circ} \mathrm{C}$ under fluorescence white light (fluence rate of 40 $60 \mu \mathrm{mol} \mathrm{m}^{-2} \mathrm{~s}^{-1}$ ) with a $16 \mathrm{~h}$ light/ $8 \mathrm{~h}$ dark photoperiod. For red-light treatments, seedlings were grown under continuous red light (fluence rate of $35 \mu \mathrm{mol} \mathrm{m} \mathrm{m}^{-2} \mathrm{~s}^{-1}$ provided by LEDs). For dark-grown seedlings, plates were wrapped in several layers of aluminium foil. Plates were placed in a vertical orientation and scanned for hypocotyl length using the ImageJ software (http://rsb.info.nih.gov/ij). For PAC treatment, seeds were transferred to plates with the inhibitor, after induction of germination. At least 20 seedlings were measured for each set of experiments.

Plant transformation. A complementary DNA fragment including the fulllength ORF of PIF4 was amplified with primers PIF4-GFPf and PIF4-GFPr, cloned into the $p E N T R / D-T O P O$ vector (Invitrogen) and inserted into the pK7FWG2 binary vector (http://www.psb.ugent.be/gateway/) with the LR clonase (Invitrogen). This binary construct was introduced into the pGV3101 strain of Agrobacterium and transformed into wild-type Col- 0 and phyB Arabidopsis plants, using the floral dip transformation method $^{33}$. Transformants were selected on kanamycin-containing medium and analysed for PIF4-GFP nuclear fluorescence. Homozygous lines were selected for strong expressers.

GA, PAC and MG132 treatments. GA $\mathrm{G}_{3}$ (GA) and paclobutrazol (PAC) stocks were freshly prepared in ethanol. The proteasome inhibitor MG132 was dissolved in DMSO. For MG132 treatment, seedlings were pre-incubated for $2 \mathrm{~h}$ in a $100 \mu \mathrm{M}$ solution of the inhibitor. PAC and GA treatments were performed at $0.1 \mu \mathrm{M}$ and $50 \mu \mathrm{M}$, respectively, unless indicated. Sensitivity to the inhibitor was calculated as the inverted ratio of the reduction in hypocotyl length observed for seedlings grown in $0.1 \mu \mathrm{M}$ PAC relative to the reduction observed in Col- 0 plants $(100 \%)$. Values are the mean of three independent experiments. GA sensitivity was calculated as the ratio of the increase in hypocotyl length of seedlings in $5.0 \mu \mathrm{M} \mathrm{GA}_{3}$ and referenced to wild-type Col-0 seedlings, as before.

Yeast two-hybrid assays. DELLA repressors are encoded in potato by at least two genes, the transcript represented by the expressed sequence tag TC113247 being the most abundantly expressed in vegetative tissues. The full-length open reading frame for this DELLA protein was amplified using primers FPG1 and FPG2 and inserted in frame with the GAL4-BD into the pBridge vector (Clontech). To avoid the auto-activation activity associated with this full-length construct, $\mathrm{N}$-end (residues 1 to 188), F1 (residues 1 to 362), M5 (residues 188 to 588) and Cter (residues 362 to 588) constructs fused to the GAL4-BD were obtained by digestion at the unique SpeI and EcoRI restriction sites. Plasmids containing these constructs, the empty pBridge vector $(p G B)$ or a $p 53-G A L 4 B D$ fusion in the $p G B K T 7$ vector (control) were transformed into AH109 yeast cells and plated on SD - Trp and SD - Trp - Ade - His plates, to test for basal activation (Supplementary Fig. 1). Yeast strains containing the F1, M5 or Cter constructs gave the lowest background activity and were used as bait. Yeast cells containing these constructs were transformed with a leaf potato complementary DNA library fused to the GAL4-AD in the $P A D-G A L 4$ vector (Stratagene) and $1-3 \times 10^{6}$ independent transformants selected on $\mathrm{SD}-\mathrm{Leu}-\mathrm{Trp}-\mathrm{Ade}-\mathrm{His}$ for positive interaction.

A DNA fragment corresponding to the Arabidopsis PIF4 full-length protein was generated by PCR amplification with primers PIF4yf and PIF4yr and inserted into the EcoRI site of the yeast $p G B K T 7$ and $p G A D T 7$ vectors. Constructs for the Arabidopsis PIF3-GAL4BD and PIF3-GAL4AD fusions were provided by P. Quail.

Deletion constructs for the DELLA and PIF4 proteins were obtained by inverse PCR reaction on the RGA-BD and PIF4-AD constructs in the $p B r i d g e$ and $p A D-$ GAL4 vectors. Constructs del1RGA and del2RGA were generated using primers RGAdel 1 or RGAdel 2 and pBridBam, which introduce a BamHI restriction site at each end. To generate constructs del1PIF4, del2PIF4 and del3PIF4, the unique
XbaI site in the $p A D$-GAL4 polylinker was deleted by fill-in. This plasmid was then used as a template for inverse PCR reactions with primers PIF4dell, PIF4del2 or PIF4del3 and $p G A D X b a$, introducing an XbaI restriction site at each end. PCR products were digested either with BamHI or XbaI, religated and transformed into Escherichia coli to obtain the different constructs. These plasmids were transformed into the AH109 yeast strain and plated on SD-4 plates to assay for interaction. $\beta$-galactosidase activity was determined on liquid cultures of these transformants using the ONPG substrate and standard protocol conditions.

Constructs equivalent to the potato RGA M5 fragment for the Arabidopsis DELLA genes RGA1 (At2g01570), GAI (At1g14920), RGL1 (At1g66350) and $R G L 3$ (At5g17490) were generated by PCR amplification with the primer sets RGL1pGB-f/RGL1pGB-r, RGL3pGB-f/RGL3pGB-r, RGApGB-f/RGApGB-r and RGApGB-f/GAIpGB- $r$ (Supplementary Table 1). These fragments were inserted into the yeast $p G B K T 7$ vector using the restriction enzymes BamHI/ SalI for RGL1, SalI/PstI for RGL3 and BamHI/PstI for RGA1 and GAI.

Pull-down assays. A DNA fragment encoding the Arabidopsis RGA full-length protein was obtained by PCR amplification with primers RGAgst-f and RGAgst$r$ and cloned into the BamHI site of the $p Z E X$ vector, to obtain an in-frame fusion with the GST coding region. The RGA-pZEX and empty $p Z E X$ vector were transformed into the E. coli strain BL21(DE3)pLysS (Stratagene) and cultures of these cells grown at $37^{\circ} \mathrm{C}$ to a $D_{600}=0.8$. Expression of the GST proteins was induced with $1 \mathrm{mM} \mathrm{IPTG}$, for 3 additional hours at $30^{\circ} \mathrm{C}$, and protein extraction and binding to gluthathione-Sepharose beads (Clontech) was performed according to the protocol supplied by the manufacturer.

The PIF4, PIF3 and carboxy-terminal phyA constructs in the $p G B K T 7$ vector were used as templates for in vitro transcription/translation, in the presence of ${ }^{35} \mathrm{~S}$-methionine, using the TnT system (Promega). Plasmid DNA $(1 \mu \mathrm{g})$ was used in each reaction. For pull-down, $10 \mu \mathrm{l}$ of the translation reactions were incubated for $30 \mathrm{~min}$ with the RGA-GST and GST beads ( $1 \mu \mathrm{g}$ of protein bound to the beads) in PBS, $0.05 \%$ tergitol, $10 \%$ glycerol. Beads were thoroughly washed, resuspended in $2 \times$ loading buffer and analysed by SDS-PAGE for protein binding.

Transient expression assays. DNA fragments encoding the Arabidopsis fulllength PIF4 and RGA proteins, and the $\triangle$ RGA and del1RGA deletions were obtained by PCR amplification with primer pairs PIF4yf and PIF4yr, RGAgst-f and RGAgst-r, $\Delta$ RGA-f and RGAgast-r and del1RGA-f and RGAgst-r, and inserted into the polilynker EcoRI or BamHI sites of the pJIT60 vector, under the control of the $2 \times 35 S$ promoter. A G-box motif fragment reported by gelshift assays to be a target element for PIF4 recognition ${ }^{34}$ was fused to the GUS reporter gene and used as a reporter construct. We were not able to detect PIF4mediated stimulation of this reporter construct, indicating that additional nucleotide sequences surrounding the CACGTG motif might be required for efficient activation. To search for a promoter element suitable to be used in these assays, preliminary RNA profiling experiments of 35S-PIF4 and pif4 mutants were performed, hence identifying LTP3 (At5g59320) as a strong upregulated transcript in 35S-PIF4 seedlings. The promoter region for this gene was amplified using primers LT3p-f and LTP3p-r and inserted into the EcoRI and BamHI sites of the $p G U S$ vector to obtain the LTP3-GUS reporter construct. The $2 \times 35 S$ luciferase fusion was used as an internal control. Arabidopsis cells were spread on filter paper and incubated overnight on LT87 medium $\left(4.4 \mathrm{gl}^{-1}\right.$ Murashige and Skoog salts + vitamins, $0.5 \mathrm{gl}^{-1} \mathrm{MES}, 0.5 \mathrm{gl}^{-1} \mathrm{NAA}, 30 \mathrm{gl}{ }^{-1}$ sucrose, $8 \mathrm{gl}^{-1}$ agar). Two hours before bombardment, filters were transferred to LT87 medium with $200 \mathrm{mM}$ mannitol to induce vacuole retraction. DNA precipitation and particle bombardment was performed using a helium-driven particle accelerator (PDS-1000; Bio-Rad), according to the manufacturer's instructions. Cells were transformed with $0.5 \mu \mathrm{g}$ of the LTP3-GUS reporter plasmid $+0.5 \mu \mathrm{g}$ of the $2 \times 35 S-L U C$ internal standard, and either $2 \mu \mathrm{g}$ of the pJIT60 empty vector (LTP3), $1 \mu \mathrm{g}$ of the $2 \times 35$ S-PIF4 effector construct $+1 \mu \mathrm{g}$ pJIT60 (PIF4), or $1 \mu \mathrm{g}$ of each $2 \times 35 S-P I F 4$ and $2 \times 35 S-R G A, 2 \times 35 S-\triangle R G A$ or $2 \times 35 S-d e l 1 R G A$ effector constructs (PIF4 + RGA, PIF4 + $\triangle$ RGA, PIF4 + del1RGA). Filters were transferred to fresh LT87 plates $(-\mathrm{GA})$ or to LT87 plates $+50 \mu \mathrm{M} \mathrm{GA}_{3}(+\mathrm{GA})$ and incubated for $16 \mathrm{~h}$. Cells were extracted in the cell lysis buffer provided in the Luciferase Assay System kit (Promega) and cleared by centrifugation at 12,000 g for $5 \mathrm{~min}$. LUC activity was determined according to the kit and GUS activity determined by fluorometric assay ${ }^{35}$. LTP3 promoter activity was calculated as the ratio of GUS to LUC activity in each sample. Four replica plates were used for each treatment.

RNA extractions and reverse transcriptase (RT)-PCR. For gene expression analyses, total RNA was extracted from 4-day-old seedlings using the guanidine- $\mathrm{HCl}$ method $^{36}$ and subsequently treated with DNaseI (Roche) before cleaning through Qiagen RNeasy Mini columns (Qiagen). RNA ( $1 \mu \mathrm{g})$ was used for first-strand cDNA synthesis using the High-Capacity cDNA Archive Kit (Applied Biosystems) and $1.0 \mu \mathrm{l}$ of this reaction was used as template for PCR 
amplification. Primer sets AtLTP3-f/AtLTP3-r, Atexpan-f/Atexpan-r and Atactin8-f/Atactin8-r were used for amplification of the LTP3 (At5g59320), $\beta$-expansin (At2g20750) and actin-8 (At1g49240) transcripts.

Bimolecular fluorescence complementation (BiFC) assays. Full-length open reading frame sequences for the Arabidopsis PIF4 and RGA proteins were amplified with primers PIF4YFP-f/ PIF4YFP-r and RGAYFP-f/ RGAYFP-r, respectively. The PCR products were cloned into the $p E N T R / D-T O P O$ vector (Invitrogen) and inserted by LR-reaction (Invitrogen) into the binary pBiFC vectors (F. Parcy) containing the amino- or C-terminal fragments of the eYFP fluorescent protein $\left(\mathrm{eYFP}^{\mathrm{N}}\right.$ and $\left.\mathrm{eYFP}^{\mathrm{C}}\right)$. All eight possible pairwise combinations of these constructs were transformed into Agrobacterium tumefaciens and co-infiltrated into the abaxial surface of 2-3-week-old Nicothiana benthamiana plants as described ${ }^{37}$. The 19 protein of tomato bushy stunt virus was used to suppress gene silencing. Agrobacterium strains containing the pBiFC constructs and the p19 silencing plasmid were at a $D_{600}$ ratio of 0.7:0.7:1 for infiltration. Fluorescence was visualized in epidermal cell layers of the leaves after 2 days of infiltration, using a Leica DMR fluorescent microscope. Leaves were incubated with $1 \mu \mathrm{g} \mathrm{ml}^{-1} 4^{\prime}$,6-diamidino-2-phenylindole (DAPI) for nuclei staining. PIF4/RGA-GFP co-immunoprecipitations. Co-immunoprecipitation studies of the RGA and PIF4 proteins were performed on 7-day-old RGA-GFP-RGA transgenic seedlings ${ }^{10}$ grown under white light. Seedlings were either incubated in darkness or transferred to PAC- or GA-containing medium and incubated for 12 additional hours before extraction. Immunoprecipitation of the GFP-RGA protein was performed at $4{ }^{\circ} \mathrm{C}$ for $6 \mathrm{~h}$, using an anti-GFP antibody (Santa Cruz Biotechnology) in a buffer containing $50 \mathrm{mM}$ Tris- $\mathrm{HCl}, \mathrm{pH}$ 7.5, $150 \mathrm{mM} \mathrm{NaCl}$, $0.5 \%$ Triton X-100, PMSF (1 mM) and protease inhibitors (Sigma). Protein G agarose (Sigma) was used to precipitate the immunoprotein complexes. PIF4 detection was performed with an anti-PIF4 antibody.

Chromatin immunoprecipitations and PCR amplifications. Chromatin immunoprecipitation assays were performed as described previously ${ }^{29}$. PIF4HA seedlings ${ }^{20}$ were grown on GM medium for 6 days under continuous red light and then were transferred to plates containing either GA or PAC and kept overnight in dark. Seedlings (1.5-2 g) and $40 \mu \mathrm{l}$ of the anti-HA Affinity Matrix (Roche) were used for chromatin immunoprecipitation. Precipitated DNA was dissolved in $50 \mu \mathrm{l}$ of TE, and $0.5 \mu \mathrm{l}$ was used for PCR amplification using the primers listed in Supplementary Table 1. PCR conditions were as follows: $94{ }^{\circ} \mathrm{C}$ for $2 \mathrm{~min}, 35$ cycles of $94^{\circ} \mathrm{C}$ for $15 \mathrm{~s}, 55^{\circ} \mathrm{C}$ for $30 \mathrm{~s}$ and $72^{\circ} \mathrm{C}$ for $30 \mathrm{~s}$, followed by $72{ }^{\circ} \mathrm{C}$ for $7 \mathrm{~min}$. Sonicated input DNA $(0.3 \%)$ was used for a quantitative control. Western blot analyses were performed to quantify the amount of recovered PIF4 protein after chromatin immunoprecipitation. Blots were immunodetected with an anti-HA peroxidase High Affinity antibody (Roche).

Microscopy. Protein stability assays were performed using the Radiance 2100 (Bio-Rad) Laser Scanning System coupled to a Zeiss Axiovert 200 microscope.
For GFP and chlorophyll excitation, an argon ion laser at 488-nm wavelength and a red diode at $637 \mathrm{~nm}$ were employed, respectively. The combination of filters used was: 560 DCLPXR beam splitter and HQ 515/30 emission filter for GFP, and HQ 660LP for chlorophyll detection. The images were sequentially taken employing the LaserSharp v5.0 software (Bio-Rad) and merged using the LaserPix v.4 image software (Bio-Rad).

Gel-shift assay. The oligonucleotides pLTP-WTf/r and pLTP-MUTf/r were used to generate the LTP3 probes and specific competitors used in EMSA assays. Oligonucleotides were annealed in $5 \times \mathrm{M}$ restriction enzyme buffer and endlabelled by fill-in with Klenow. For protein extracts, N. benthamiana leaves were infiltrated with the Agrobacterium strains for the 35S-PIF4-HA (PIF4) and 35S$R G A-G F P(\mathrm{RGA})$ constructs or with a 1:1 mixture (PIF4 + RGA) of these strains and with the p19 construct as described above. Leaf extracts were obtained by homogenization in high salt buffer (20 mM HEPES, pH 7.9, $0.5 \mathrm{M} \mathrm{KCl}, 1 \mathrm{mM}$ EDTA, $1 \mathrm{mM} \mathrm{MgCl}_{2}, 0.5 \%$ nonidet P-40, $1 \mathrm{mM}$ DTT, + protease inhibitor), clearing by centrifugation and subsequent dialysis against $1 \times \mathrm{BB}(20 \mathrm{mM}$ HEPES, pH 7.9, 0.1 M KCl, $1 \mathrm{mM}$ EDTA, 0.05\% nonidet P-40, $0.5 \mathrm{mM}$ DTT, $10 \%$ glycerol). The presence of equivalent amounts of PIF4 and RGA proteins in the extracts was assessed by western blot detection with anti-HA (Roche) and anti-GFP (Santa Cruz) antibodies. Increasing amounts of these extracts (5.0, $10.0,15.0 \mu \mathrm{l})$ were used for the EMSA reaction. Extracts were incubated for $15 \mathrm{~min}$ at room temperature with the labelled probe and $100 \mathrm{ng}$ poly (dI-dIC) in $20 \mu \mathrm{l} 1 \times \mathrm{BB}$ and separated by $6 \%$ PAGE in $0.5 \times$ TBE. A 100 -fold excess of wild-type (WT) and mutant (MUT) annealed oligonucleotides was used for specific competition.

31. Reed, J. W., Nagpal, P., Poole, D. S., Furuya, M. \& Chory, J. Mutations in the gene for the red/far-red light receptor phytochrome B alter cell elongation and physiological responses throughout Arabidopsis development. Plant Cell 5, 147-157 (1993).

32. Huq, E., Kang, Y., Halliday, K. J., Qin, M. \& Quail, P. H. SRL1: a new locus specific to the phyB-signaling pathway in Arabidopsis. Plant J. 23, 461-470 (2000).

33. Clough, S. J. \& Bent, A. F. Floral dip: a simplified method for Agrobacterium-mediated transformation of Arabidopsis thaliana. Plant J. 16, 735-743 (1998).

34. Martinez-Garcia, J. F., Huq, E. \& Quail, P. H. Direct targeting of light signals to a promoter element-bound transcription factor. Science $288,859-863$ (2000).

35. Jefferson, R. A. The GUS reporter gene system. Nature 342, 837-838 (1989).

36. Logemann, J., Schell, J. \& Willmitzer, L. Improved method for the isolation of RNA from plant tissues. Anal. Biochem. 163, 16-20 (1987).

37. Voinnet, O., Rivas, S., Mestre, P. \& Baulcombe, D. An enhanced transient expression system in plants based on suppression of gene silencing by the p19 protein of tomato bushy stunt virus. Plant J. 33, 949-956 (2003). 